\title{
Controlled growth of GaN nanowires by pulsed metalorganic chemical vapor deposition
}

\author{
G. Kipshidze, ${ }^{\text {a) }}$ B. Yavich, A. Chandolu, J. Yun, V. Kuryatkov, I. Ahmad, D. Aurongzeb, \\ M. Holtz, and H. Temkin \\ Nano Tech Center, Texas Tech University, Lubbock, Texas 79401
}

(Received 9 September 2004; accepted 10 November 2004; published online 11 January 2005)

Controlled and reproducible growth of $\mathrm{GaN}$ nanowires is demonstrated by pulsed low-pressure metalorganic chemical vapor deposition. Using self-assembled Ni nanodots as nucleation sites on (0001) sapphire substrates we obtain nanowires of wurtzite-phase GaN with hexagonal cross sections, diameters of about $100 \mathrm{~nm}$, and well-controlled length. The nanowires are highly oriented and perpendicular to the growth surface. The wires have excellent structural and optical properties, as determined by $\mathrm{x}$-ray diffraction, cathodoluminescence, and Raman scattering. The x-ray measurements show that the nanowires are under a complex strain state consistent with a superposition of hydrostatic and biaxial components. (C) 2005 American Institute of Physics.

[DOI: $10.1063 / 1.1850188$ ]

Semiconductor nanowires are quasi-one-dimensional structures exhibiting unique electrical and optical properties. ${ }^{1,2}$ Considerable effort has been devoted to the synthesis of nanowires of $\mathrm{GaN}$, a technologically important large band gap semiconductor. Wire preparation by plasmaassisted molecular-beam epitaxy, ${ }^{3}$ laser ablation of $\mathrm{GaN}$ containing targets, ${ }^{4}$ reaction of $\mathrm{Ga} / \mathrm{Ga}_{2} \mathrm{O}_{3}$ mixtures with $\mathrm{NH}_{3}$ in anodized alumina templates, ${ }^{5}$ direct reaction of $\mathrm{Ga}$ with $\mathrm{NH}_{3}$ in a tube furnace, ${ }^{6}$ and chemical vapor deposition from $\mathrm{Ga}$-containing precursors and $\mathrm{NH}_{3}$ on catalyst-coated substrates $^{7-9}$ have all been reported. The wire preparation is usually based on the vapor-liquid-solid (VLS) growth mechanism ${ }^{10,11}$ with the use of catalytic nanoparticles of In, $\mathrm{Au}, \mathrm{Fe}, \mathrm{Ni}$, or $\mathrm{Co}^{4,9}$ Recent interest has focused on synthesis of $\mathrm{GaN}$ nanowires by metalorganic chemical vapor deposition (MOCVD). ${ }^{12}$

In this work the nanowire samples were grown by lowpressure MOCVD in a close-coupled showerhead reactor. All growth runs were carried out at 30 Torr, using trimethylgallium (TMG) and ammonia with $\mathrm{N}_{2}$ as gas carrier. The TMG flux was varied in the range of $2-10 \mu \mathrm{mol} / \mathrm{min}$ and $500 \mathrm{sccm} \mathrm{NH}_{3}$ flow was used. The growth was nucleated from self-assembled catalytic islands of Ni that become supersaturated with $\mathrm{Ga}$ and active nitrogen supplied from the gas phase. Once nucleated, growth of GaN nanowires occurs at the solid-liquid interface. For this purpose, a layer of $\mathrm{Ni}$, 2-5 $\mathrm{nm}$ thick, was deposited by electron beam evaporation on (0001) oriented sapphire substrates. Formation of nanodispersed $\mathrm{Ni}$ islands was accomplished by heating the wafer at $830{ }^{\circ} \mathrm{C}$ for $5 \mathrm{~min}$, under nitrogen flow, in the epitaxial reactor. This procedure results in formation of Ni nanodroplets of approximately $100 \mathrm{~nm}$ in diameter. VLS growth of $\mathrm{GaN}$ nanowires was carried out at a temperature of $700{ }^{\circ} \mathrm{C}$.

In the conventional MOCVD growth both TMG and ammonia are introduced simultaneously into the reactor. However, this does not result in reproducible VLS growth of GaN nanowires. Instead, formation of three-dimensional polycrystalline islands of $\mathrm{GaN}$, with very poor selectivity with respect to Ni droplets, was observed. We conclude that in the standard process the VLS mechanism is overwhelmed by

a)Electronic mail: gela.kipshidze@ttu.edu parasitic gas-phase reactions between TMG and $\mathrm{NH}_{3}$. Such gas-phase reactions generate nanoparticles of $\mathrm{GaN}$ above the wafer, ${ }^{13}$ which then deposit randomly on the surface. For that reason we experimented with pulsed MOCVD mode, which efficiently reduces, or suppresses, gas-phase reactions between precursors. ${ }^{14,15}$ In this growth mode we obtain reproducible and controllable preparation of nanowires of $\mathrm{GaN}$. The best results were obtained using 2-s-long pulses of $\mathrm{NH}_{3}$, with a 2-s-long delay between subsequent pulses of TMG and $\mathrm{NH}_{3}$. The growth rate was controlled by the pulse length of TMG. With the TMG pulse length of $2 \mathrm{~s}$, while keeping other time intervals fixed, the nanowires grow at about 8 monolayers per pulse or $\sim 1 \mu \mathrm{m} / \mathrm{h}$. The final length of the GaN nanowires was controlled by the total number of TMG pulses. Most of the wires discussed here were prepared with the diameter of $\sim 100 \mathrm{~nm}$. The growth is reproducible from run to run. With the same thickness of $\mathrm{Ni}$ and same growth conditions wire diameter, orientation, morphology, and growth rate remain constant.

Figure 1 shows a representative SEM image of GaN nanowires. These nanowires grow perpendicular to the substrate, with fairly constant diameters, smooth sidewalls, and hexagonal cross sections, as shown in inset (a) of Fig. 1 and Fig. 2. The nanowires grow selectively from islands of the

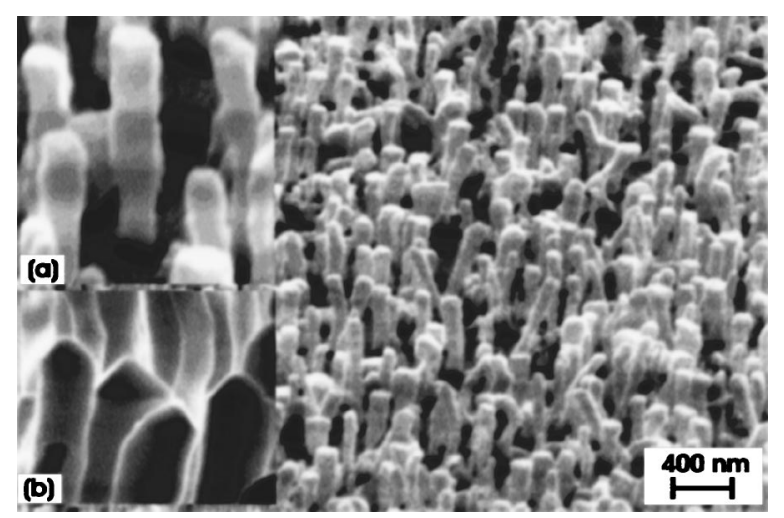

FIG. 1. SEM image of GaN nanowires showing general sample morphology. Image obtained with the stage tilted at $30^{\circ}$; (a) higher magnification image of an individual wire $100 \mathrm{~nm}$ in diameter and (b) metal caps on GaN nanowires. This sample was grown at $750{ }^{\circ} \mathrm{C}$ using fluxes of $5.6 \mu \mathrm{mol} / \mathrm{min}$ of TMG and $500 \mathrm{sccm}$ of ammonia and $\mathrm{N}_{2}$ as carrier gas. 
TABLE I. Stress parameters of GaN nanowires.

\begin{tabular}{|c|c|c|c|c|c|c|c|c|}
\hline \multirow[b]{2}{*}{ Sample } & \multicolumn{2}{|c|}{ Lattice parameter } & \multicolumn{2}{|c|}{ Strain $\left(\times 10^{-4}\right)$} & \multicolumn{2}{|c|}{ Stress (GPa) } & \multicolumn{2}{|c|}{ Raman shift $\left(\mathrm{cm}^{-1}\right)$} \\
\hline & $a(\AA)$ & $c(\AA)$ & $\varepsilon_{a}$ & $\varepsilon_{c}$ & Hydrostatic & Biaxial & Meas. & Calc. \\
\hline \multicolumn{9}{|l|}{$\mathrm{GaN}$} \\
\hline reference & 3.1892 & 5.1855 & $\cdots$ & $\cdots$ & $\cdots$ & $\cdots$ & $\cdots$ & $\cdots$ \\
\hline$L=1.5 \mu \mathrm{m}$ & 3.1891 & 5.1895 & -0.3 & 7.7 & -0.30 & -0.24 & $-0.7 \pm 0.2$ & -0.48 \\
\hline$L=0.8 \mu \mathrm{m}$ & 3.1897 & 5.1874 & 1.6 & 3.7 & -0.17 & -0.06 & $-0.8 \pm 0.2$ & -0.55 \\
\hline
\end{tabular}

metal catalyst and no deposits of GaN could be seen between the wires. A slight increase in the diameter of the growing wire, visible in Fig. 1(a), may be indicative of sidewall growth. Characteristic metal caps at nanowires tips, shown in the inset (b) of Fig. 1, confirm the VLS mechanism of their formation. The shape of the caps is typical of Ni nanodots formed in high temperature, $830{ }^{\circ} \mathrm{C}$, anneals. These caps are easily removed with a wet etch. Formation of wires with triangular cross sections ${ }^{12}$ was not observed.

XRD measurements were carried out to elucidate crystal structure of GaN nanowires. Symmetric $2 \theta-\omega$ scans carried on a sample of $\sim 1-\mu \mathrm{m}$-long nanowires over a wide angular range show only the (0002) and (0004) diffraction peaks of hexagonal $\mathrm{GaN}$ at $34.56^{\circ}$ and $72.917^{\circ}$, respectively. The data clearly confirms the $c$-axis growth orientation and hexagonal nature of the wires. Symmetric reciprocal space map obtained for the (0002) reflection of hexagonal GaN shows that the nanowires are under uniform strain.

Nanowires of GaN grown by pulsed epitaxy are sufficiently uniform, in both length and diameter, to measure the $a$ and $c$ lattice constants. These were obtained from the measurements of symmetric (0002) and asymmetric (112 4$)$ reflections. The resulting lattice constants are listed in Table I for selected samples with $\sim 100$-nm-diam wires of different lengths $(L)$, together with the lattice constants of relaxed $\mathrm{GaN}$ used as a reference. These measurements demonstrate the presence of strain in nanowires of GaN.

Optical properties of GaN layers were characterized by CL measurements at room temperature. Samples were excited with an electron beam with the energy of $1-9 \mathrm{keV}$, at a current of $100 \mu \mathrm{A}$, with the excitation power density between 1.0 and $4.5 \mathrm{~W} / \mathrm{cm}^{2}$. The spectra, shown in Fig. 2, exhibit a single peak at $3.36 \mathrm{eV}(\sim 369 \mathrm{~nm})$ which we attribute to band-edge-related emission. This peak is redshifted, by $\sim 70 \mathrm{meV}$, with respect the band edge of hexagonal GaN. The slight redshift can be attributed to strain in $\mathrm{GaN}$ nanowires, as discussed in the following. Deep level $\mathrm{CL}$ emission was not observed. The inset shows a high magnification SEM image of a single nanowire confirming its hexagonal symmetry.

As seen in Table I both the $a$ and $c$ lattice constants of our GaN nanowires differ from those of the reference sample, indicating that the wires are strained. Uniform layers of $\mathrm{GaN}$ on sapphire are usually under compressive biaxial stress, resulting in $\varepsilon_{a}<0$ and $\varepsilon_{c}>0$. This is not the case of all nanowire samples studied here. In addition, the values of Poisson ratio evaluated from our measurements are not consistent with the expected value of $0.23 \pm 0.06$ for $\mathrm{GaN}$ under compressive biaxial strain. ${ }^{16}$ This suggests a more complex strain state, a combination of hydrostatic and biaxial strains according to $\sigma=C \varepsilon$, where $\sigma$ is the stress tensor, $C$ is the Downloaded 11 Jan 2005 to 129.118 .119 .122 . Redistribution subje elastic compliance tensor, ${ }^{17}$ and $\varepsilon$ is the associated strain tensor. In our analysis, we assume a diagonal strain tensor

$$
\varepsilon=\left[\begin{array}{lll}
\varepsilon_{a} & & \\
& \varepsilon_{a} & \\
& & \varepsilon_{c}
\end{array}\right]
$$

with the associated stress tensor

$$
\sigma=-P\left[\begin{array}{lll}
1 & & \\
& 1 & \\
& & 1
\end{array}\right]+\sigma_{b}\left[\begin{array}{lll}
1 & & \\
& 1 & \\
& & 0
\end{array}\right],
$$

where $P$ is the effective hydrostatic pressure and $\sigma_{b}$ is the biaxial component of stress. This analysis allows us to determine both unknown components of the stress tensor from the two experimentally determined strains $\varepsilon_{a}$ and $\varepsilon_{c}$. Results of Table I illustrate the range of stress values determined for GaN nanowires with different length. Externally applied hydrostatic pressure is always compressive. Hence, $P<0$ indicates a tensile internal strain. Clearly, the hydrostatic component is significant. Such component is occasionally present in uniform layers of $\mathrm{GaN}$ and attributed to point defects. ${ }^{16,18,19}$ Following the approach of Ref. 16, it is possible to estimate the concentrations of likely point defects responsible for this internal strain. Nickel is a plausible candidate, since it is possible that small amounts are incorporated into the growing $\mathrm{GaN}$ nanowires forming $\mathrm{Ni}-\mathrm{Ga}$ intermetallics. A straightforward calculation would suggest $\mathrm{Ni}$ concentrations as high as $0.1 \%$. This high value seems unlikely, since we do not detect any Ni-Ga compounds in our wide angle x-ray diffraction measurements. In addition, high defect concentrations, which generally quench band-gap-related luminescence, are not consistent with the CL spectra of our samples. Internal strain driven by surface relaxation has been proposed recently to account for stiffening of nanoparticles. ${ }^{20}$ Inclusion of such a mechanism, unique to nanostructures, may be necessary to account for the strain measured in our nanowires.

Biaxial stress in epitaxial GaN layers grown on sapphire stems from residual and thermal stresses. Values are typically on the order of $-0.5 \mathrm{GPa}^{21}$ The maximum thermal stress calculated for ideal GaN layers grown on sapphire at $700{ }^{\circ} \mathrm{C}$ is $\sim-1 \mathrm{GPa}^{22,23}$ In comparison, the biaxial stress term for our nanowires varies from negligibly small, $-0.06 \mathrm{GPa}$ in wires with the length of $0.8 \mu \mathrm{m}$, to $-0.24 \mathrm{GPa}$ in $1.5-\mu \mathrm{m}$-long wires. Thus, our short nanowires show almost complete relaxation.

Based on the stress states determined from our x-ray measurements, we can also estimate the expected shift in the $\mathrm{GaN}$ energy gap for our nanowires. Using hydrostatic and uniaxial coefficients from Refs. 24 and 25 we estimate this to AlP license or copyright, see http://apl.aip.org/apl/copyright.jsp 


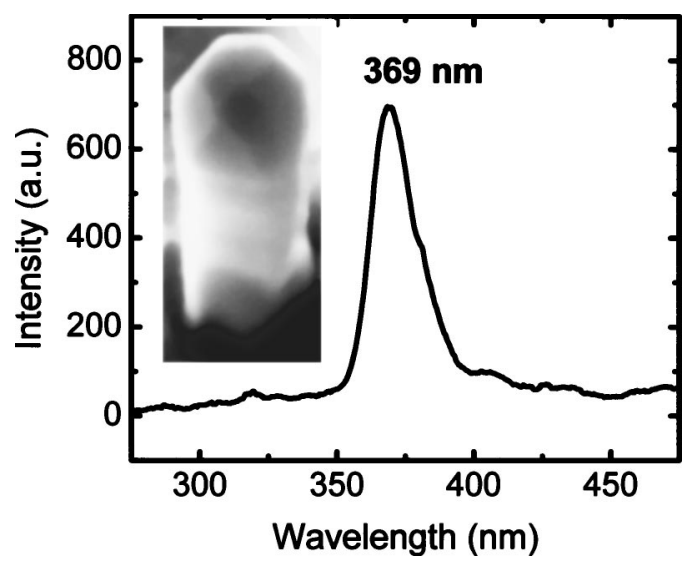

FIG. 2. Room temperature cathodoluminescence spectrum of 100-nm-diam GaN nanowires grown by pulsed MOCVD. Inset shows a high magnification SEM image of a single hexagonal nanowire.

redshift to be $30-40 \mathrm{meV}$. This is in reasonable agreement with what we report above for our CL measurements, without taking into account further factors such as the Stokes shift and shallow defect states, each of which would produce a redshift.

Additional information on the stress state of our nanowires is obtained using Raman spectroscopy. Figure 3 shows a representative room temperature Raman spectrum of the 0.8 - $\mu \mathrm{m}$-long nanowire sample (see Table I). The spectrum shows two lines $-E_{2}^{2}$, which is symmetry allowed in backscattering along the wurtzite $c$ axis, and a weak symmetry forbidden $A_{1}(\mathrm{TO})$. The latter is most likely seen due to right angle scattering occurring when the incident light enters the nanowires sidewalls, as illustrated in the inset of Fig. 3. The narrow linewidth of the $E_{2}^{2}$ phonon, $\sim 6 \mathrm{~cm}^{-1}$, is comparable to that obtained on high quality epitaxial GaN, confirming high crystal quality of the nanowires. The position of the strong $E_{2}^{2}$ line is commonly used to determine stress in epitaxial GaN. Taking the stress values listed in Table I and known Raman shifts for hydrostatic pressure $\left(4.24 \mathrm{~cm}^{-1} / \mathrm{GPa}\right)^{26}$ and biaxial stress $\left(3.24 \mathrm{~cm}^{-1} / \mathrm{GPa}\right)^{22}$ we

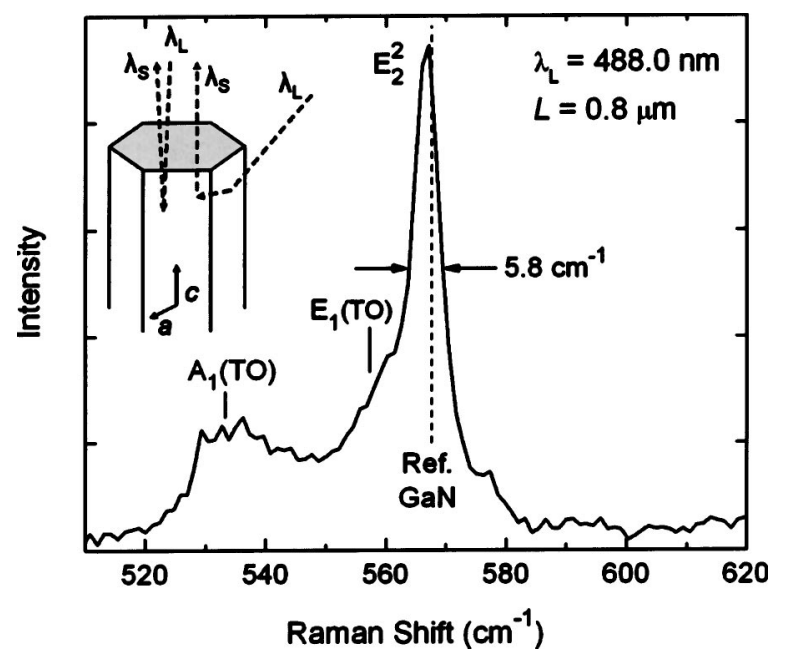

FIG. 3. Room temperature Raman spectra of GaN nanowires. The inset depicts the mechanism by which both backscattering and right angle scattering is possible, with incident (scattered) wavelength denoted as $\lambda_{L}\left(\lambda_{S}\right)$. calculate the expected Raman shift of the $E_{2}^{2}$ phonon. The experimental and calculated values of the Raman shift are shown in Table I. Good agreement seen in both sets of values supports our interpretation of the nanowires ${ }^{1}$ stress discussed earlier.

In summary, we report reproducible growth of GaN nanowires by pulsed MOCVD from Ni nanodots. X-ray diffraction and optical measurements confirm the wurtzite structure, $c$-axis growth orientation, and excellent structural and optical properties. The stress state in the wires is interpreted as a combination of hydrostatic and biaxial, with good agreement obtained between x-ray and Raman measurements.

The authors acknowledge support for this research from the National Science Foundation (ECS-0304224, ECS0210141 and ECS-0323640) and the J. F Maddox Foundation.

${ }^{1}$ Y. Xia, P. Yang, Y. Sun, Y. Wu, B. Mayers, B. Gates, Y. Yin, F. Kim, and H. Yan, Adv. Mater. (Weinheim, Ger.) 15, 353 (2003).

${ }^{2}$ Z. Zhong, F. Qian, D. Wang, and C. M. Lieber, Nano Lett. 3, 343 (2003).

${ }^{3}$ V. V. Mamutin, Tech. Phys. Lett. 25, 741 (1999).

${ }^{4}$ X. Duan and C. Lieber, J. Am. Chem. Soc. 122, 188 (2000).

${ }^{5}$ G. Chen, L. Zhang, Y. Zhu, G. Fei, L. Li, C. Mo, and Y. Mao, Appl. Phys. Lett. 75, 2455 (1999).

${ }^{6}$ M. He, P. Zhou, S. Noor Mohammad, G. L. Harris, J. B. Halpern, R. Jacobs, W. L. Sarney, and L. Salamanca-Riba, J. Cryst. Growth 231, 357 (2001).

${ }^{7}$ S. Han, W. Jin, T. Tang, C. Li, D. Zhang, and X. Liu, J. Mater. Res. 18, 245 (2003).

${ }^{8}$ T. Y. Kim, S. H. Lee, Y. H. Mo, H. W. Shim, K. S. Nahm, E.-K. Suh, J. W. Yang, K. Y. Lim, and G. S. Park, J. Cryst. Growth 257, 97 (2003).

${ }^{9}$ G. Cheng, A. Kolmakov, Y. Zhang, M. Moskovits, R. Munden, M. Reed, G. Wang, D. Moses, and J. Zhang, Appl. Phys. Lett. 83, 1578 (2003).

${ }^{10}$ R. S. Wagner and W. C. Ellis, Appl. Phys. Lett. 4, 89 (1964).

${ }^{11}$ R. S. Wagner, W. C. Ellis, K. A. Jackson, and S. M. Arnold, J. Appl. Phys. 35, 2993 (1964)

${ }^{12}$ T. Kuykendall, P. Pauzauskie, S. Lee, Y. Zhang, J. Goldberger, and P. Yang, Nano Lett. 3, 1063 (2003).

${ }^{13}$ J. R. Creighton, W. G. Breiland, M. E. Coltrin, and R. P. Pawlowski, Appl. Phys. Lett. 81, 2626 (2002).

${ }^{14}$ M. Asif Khan, R. A. Skogman, J. M. Van Hove, D. T. Olson, and J. N. Kuznia, Appl. Phys. Lett. 60, 1366 (1992).

${ }^{15}$ J. Zhang, E. Kuokstis, Q. Fareed, H. Wang, J. Yang, G. Simin, M. Asif Khan, R. Gaska, and M. Shur, Appl. Phys. Lett. 79, 925 (2001).

${ }^{16}$ C. Kisielowski, J. Krüger, S. Ruvimov, T. Suski, J. W. Ager III, E. Jones, Z. Liliental-Weber, M. Rubin, E. R. Weber, M. D. Bremser, and R. F. Davis, Phys. Rev. B 54, 17745 (1996).

${ }^{17}$ A. F. Wright, J. Appl. Phys. 82, 2833 (1997).

${ }^{18}$ M. Katsikini, K. Papagelis, E. C. Paloura, and S. Ves, J. Appl. Phys. 94, 4389 (2003).

${ }^{19}$ M. J. Aziz, Appl. Phys. Lett. 70, 2811 (1997).

${ }^{20}$ B. Gilbert, F. Huang, H. Zhang, G. A. Waychunas, and J. F. Banfield, Science 305, 651 (2004).

${ }^{21}$ J. Keckes, J. W. Gerlach, R. Averbeck, H. Riechert, S. Bader, B. Hahn, H.-J. Lugauer, A. Lell, V. Harle, A. Wenzel, and B. Rauschenbch, Appl. Phys. Lett. 79, 4307 (2001).

${ }^{22}$ I. Ahmad, M. Holtz, N. N. Faleev, and H. Temkin, J. Appl. Phys. 95, 1692 (2004).

${ }^{23}$ H. Hiramatsu, T. Detchprohm, and I. Akasaki, Jpn. J. Appl. Phys., Part 1 32, L1528 (1993).

${ }^{24}$ P. Perlin, I. Gorczyca, N. E. Christensen, I. Grzegory, H. Teisseyre, and T. Suski, Phys. Rev. B 45, 13307 (1992).

${ }^{25}$ D. G. Zhao, S. J. Xu, M. H. Xie, S. Y. Tong, and H. Yang, Appl. Phys. Lett. 83, 677 (2003).

${ }^{26}$ A. R. Goňi, H. Siegle, K. Syassen, C. Thomsen, and J.-M. Wagner, Phys. Rev. B 64, 035205 (2001). 